\title{
A Semi-automatic Label Digitization Workflow for the Siegfried Map
}

Keywords: historical maps, vectorization, deep learning, convolutional neuronal network, label extraction

Summary: Digitizing historical maps automatically offers a multitude of challenges. This is particularly true for the case of label extraction since labels vary strongly in shape, size, orientation and type. In addition, characters may overlap with other features such as roads or hachures, which makes extraction even harder. To tackle this issue, we propose a novel semi-automatic workflow consisting of a sequence of deep learning and conventional text processing steps in conjunction with tailormade correction software. To prove its efficiency, the workflow is being applied to the Siegfried Map Series (1870-1949) covering entire Switzerland with scales $1: 25.000$ and $1: 50.000$. The workflow consists of the following steps. First, we decide for each pixel if the content is text or background. For this purpose, we use a convolutional neuronal network with the U-Net architecture which was developed for biomedical image segmentation (Ronneberger, 2015). The weights are calculated with four manually annotated map sheets as ground truth. The trained model can then be used to predict the segmentation on any other map sheet. The results are clustered with DBSCAN (Ester, Kriegel, Sander, \& Xu, 1996) to aggregate the individual pixels to letters and words. This way, each label can be localized and extracted without background. But since this is still a non-vectorized representation of the labels, we use the Google Vision API to interpret the text of each label and also search for matching entries in the Swiss Names database by Swisstopo for verification. As for most label extraction workflows, the last step consists of manually checking all labels and correcting possible mistakes. For this purpose, we modified the VGG Image Annotator to simplify the selection of the correct entry. Our framework reduces the time consumption of digitizing labels drastically by a factor of around 5. The fully automatic part (segmentation, interpretation, matching) takes around 5-10 min per sheet and the manual processing part around 1.5-2h. Compared to a fully manual digitizing process, time efficiency is not the only benefit. Also the chance of missing labels decreases strongly. A human cannot detect labels with the same accuracy as a computer algorithm. Most problems leading to more manual work occur during clustering and text recognition with the Google Vision API. Since the model is trained for maps in a flat part of German-speaking Switzerland, the algorithm performs poorer for other parts. In Alpine regions, the rock hachures are often misinterpreted as labels, leading to many false positives. French labels are often composed of several words, which are not clustered into one label by DBSCAN. Possible further work could include retraining with more diverse ground truth or extending the U-Net model so that it can also recognize and learn textual information.

\footnotetext{
${ }^{1}$ Institute of Cartography and Geoinformation, ETH Zurich, Switzerland [laumerd@ student.ethz.ch]

${ }^{2}$ Institute of Cartography and Geoinformation, ETH Zurich, Switzerland
} 


\section{Introduction}

The Siegfried Map was the official map series from Switzerland from 1870 to 1949. It is the successor of the Dufour Map which was the first official Swiss map. Some of the main differences is the change from hachures to contours and the introduction of colors. The production included highly tedious work from surveying in alpine terrain and engraving the map contents on different copper plates. The cartographers took much attention to detail and wanted the map not only to be a mere means to an end but also a work of art. Especially the meticulously drawn rock hachures helped to shape the reputation of the famous Swiss cartography style (Bleuer, 1968). The map was produced in two different scales, $1: 50.000$ in the mountainous regions and $1: 25.000$ for the lowland. It consists of around 500 different sheets, each spanning around $200 \mathrm{~km}^{2}$ for the 1:50.000 regions or $50 \mathrm{~km}^{2}$ for the $1: 25.000$ regions.

In the efforts of preserving the map and making it available for future generations, each sheet has already been scanned and georeferenced. But to use the full potential of analysis of historic data, current projects focus on the vectorization of the different map elements like buildings (Heitzler \& Hurni, 2020), contours or hydrological features. The objective of this project is part of this bigger goal and namely deals with the extraction of the labels together with their respective position. Those digitized labels will help for historical analysis and show how the toponyms changed over time due to spatial development.

Since there are around 500 sheets with multiple versions, amounting to around 4000 sheets in total, a pure manual label annotation process is considered to be unfeasible. Therefore, the main part of the process was designed to be automatic, however a final manual correction step could not be avoided.

\section{Related Work}

The interest in digital map processing started in the early 80's. One of the main reasons was the nearly exponential growth of scanned maps in numerous archives. Many disciplines in the natural and social sciences became increasingly interested in using historical maps as a primary resource (Chiang, Unlocking Textual Content from Historical Maps - Potentials and Applications, Trends, and Outlooks, 2017). Also to deeply understand the impact of environmental change, analyzing historical maps can be essential. They can provide a new dimension to the processing of geographic data by expanding from spatial to spatio-temporal analysis (Chiang, Leyk, \& Knoblock, 2014).

Label extraction of historical maps is a very difficult task. Labels can vary strongly in shape, form, size, and type. Especially characters overlapping with roads or shading make this even harder. While other features on historical maps like roads or buildings often have a more consistent structure and are easier to extract, labels can vary greatly in appearance. They can be written with different angles, bending or even different spacing.

These issues have been addressed with different approaches. Deseilligny et al. (1995) developed a rotation-invariant character recognition method to overcome the issue that pure Optical Character Recognition (OCR) likely mixed up similar characters. Frischknecht (1996) introduced a software package which extracts symbols and labels on scanned maps with a socalled knowledge-based matching method. To solve the very difficult separation process of text and background graphics, Velázquez and Levachkine (2004) developed a method called Vlines which can also handle curved labels. An OCR-based recognition algorithm using artificial 
neural networks is applied to define the coordinates, size, and orientation of the character strings. An increasing trend in image processing research is the use of deep learning algorithms. For example Wick and Puppe (2018) proposed a fully convolutional neural network for historical document segmentation of text and pictures.

\section{Methodology}

This project also follows the aforementioned trend of using deep learning algorithms. The workflow is described in Figure 1. First we use a previously trained Convolutional Neuronal Network $(\mathrm{CNN})$ to predict a pixel wise segmentation of the map into label and non-label areas (1). To determine which pixels belong to the same word, the areas are clustered using DBSCAN (2). With this information, for each word the corresponding bounding box can be extracted from the map and the background can be removed, so that the word can be read more clearly $(3+4)$. This is then fed into the Google Vision API, which is designed to read text from images (5). This is sometimes still erroneous. To refine the result, we use an existing database of the labels in the current map work. The vicinity is checked for a similar label and recorded if there is a match (6). To determine the final result, the last step is to manually review the labels and correct potential errors.

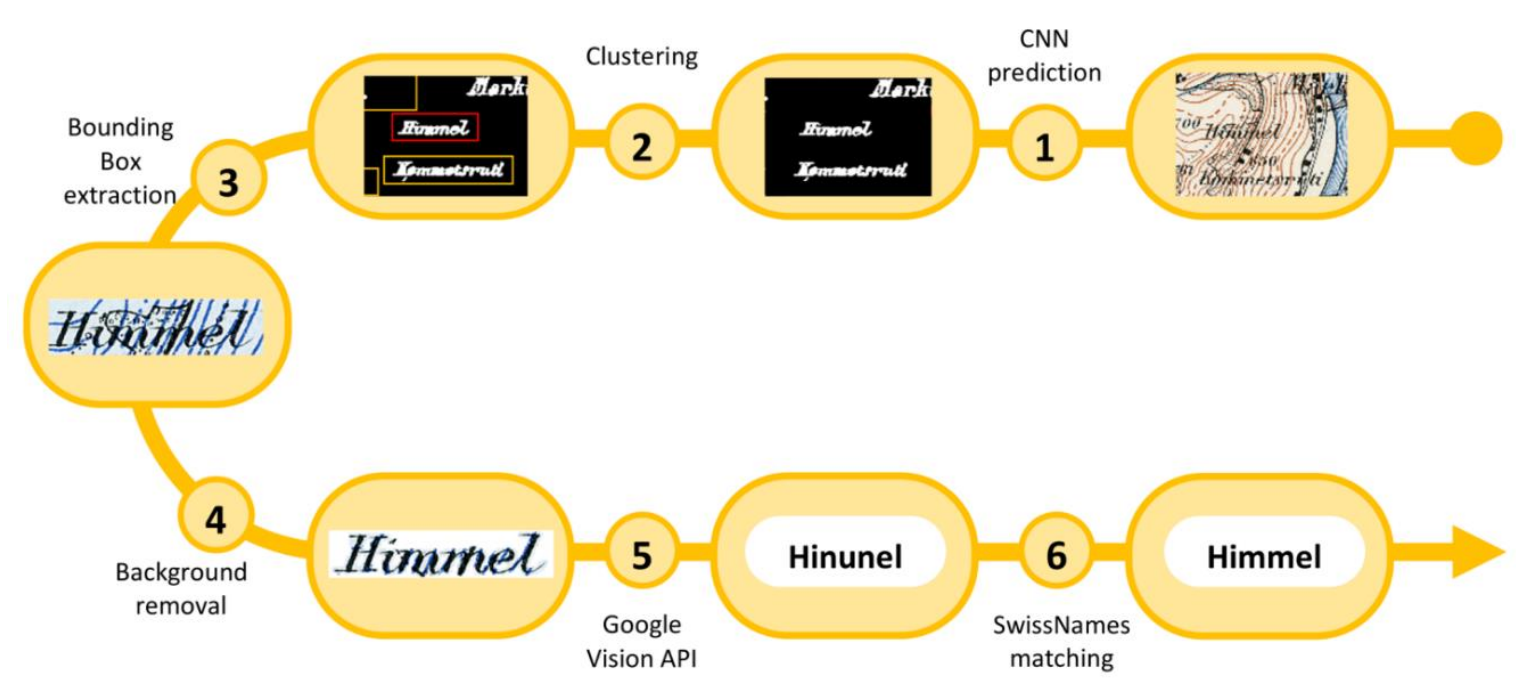

Figure 1. Automatic workflow schematics

\section{Training the Deep Learning Network}

A CNN is a deep learning algorithm which can learn to find and classify patterns on images. Several different classes of patterns can be defined for the deep learning model to look for. It takes as input the individual pixels and numerous parameters and outputs to which class each pixel most probably belongs to. The parameters are also called weights and have to be trained beforehand. This training needs ground truth data, meaning map sheets where each pixel is already labelled as text or background. With the help of this, the algorithm can adapt the weights according to the specific problem.

In this project, the ground truth was produced with cumbersome manual annotation of four sheets. Polygons were drawn over each word, following the exact shapes of the letters. This took around one workday per sheet. Of the $4800 \times 7000$ pixels, only around $2 \%$ represent text. Therefore we have a strongly unbalanced training data (see Figure 2). 
For the CNN we use the U-Net model which was proposed by Ronneberger et al. (2015) for biomedical image segmentation. As an input it takes images of $256 \times 256$ pixels. Therefore, we crop out input sheets into batches of 200x200 pixels and then up-sample them to the required $256 \times 256$ pixels. The training consists of iteratively adjusting the weights to better fit the ground truth dataset using gradient descent. The loss function is a measure of how well the model behaves in comparison to the desired values. When the loss function converges to a small enough value, the training is completed and the weights are saved for prediction on other sheets.
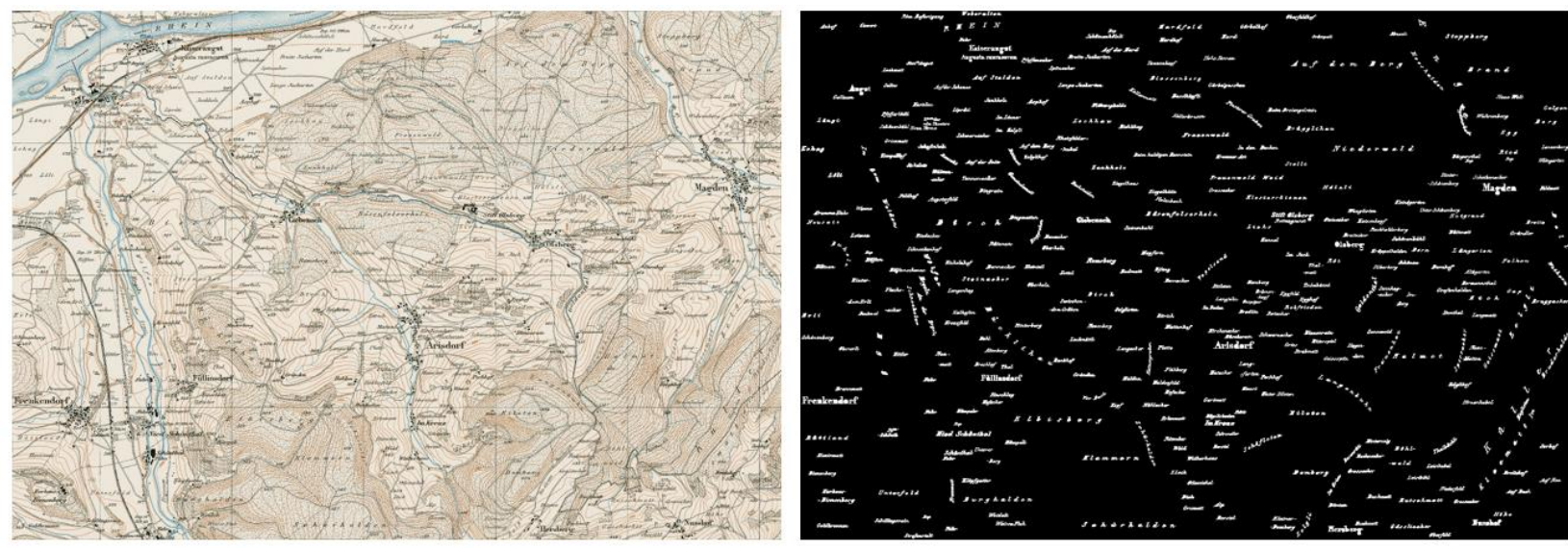

Figure 2. Training data sheet (left) and ground truth (right)

\section{CNN Prediction}

When the model is trained, it can be used to predict the segmentation of any other sheet. Before feeding the sheet into the algorithm, it is also first cropped into smaller batches in order to speed up the process. When the network finishes the pixel-wise prediction of the class, the output is a value between 0 and 1 . This indicated the likelihood of the pixel to belong to the foreground class. We defined a threshold of 0.5 to define the class for each pixel. All values above are classified as text. The last step is to recombine the small batches to the size of the input sheet.

\section{Clustering Results}

To determine which segments belong to a common label, the results of the prediction are grouped using a density based clustering algorithm called DBSCAN. It has the advantage that arbitrary shaped clusters can be found and it is also robust to outliers. This is especially helpful for maps since multiple labels of different form and size can often be found close to each other. The parameters for this clustering method were found empirically and might have to be adjusted for different types of maps. When all clusters are found, a bounding box for each cluster is defined.

\section{Google Vision and Swiss Names Matching}

After the location and segmentation of each labels is found, the next step is to understand the content of the text. For this we need a tool provided by Google Vision API (Google, 2020). It allows uploading images containing text and outputs the digitalized text. There are several pre-trained networks which work on many different text fonts and styles. 
To prepare the images to be sent to Google Vision, each label found in the clustering step is cropped using the corresponding bounding box and saved as a separate image. In order to reduce errors caused by confusing background patterns, we use the segmentation information to delete the background parts on the cropped images. This makes the text stand out and more clearly visible.

The Google Vision API does not always work correctly. Sometimes there are some wrong or extra letters. To address this issue, the last step of the automatic workflow is to compare it with an existing database. For the up-to-date maps of Switzerland there is an open-data database in vector form available, called SwissNames (Swisstopo, 2013). For each result of the API, the existing database is searched for labels in an area of $1 \mathrm{~km}^{2}$ around the result. Then the label which is most similar is chosen as the best match. As a measure of similarity, the Levenshtein distance was used (Levenshtein, 1966). Over the years the label changed significantly, but for some it can still improve the result produced by the Google Vision API. To decide which label is correct and in which cases to take the matched result from SwissNames, some manual postprocessing is needed.

\section{Manual Annotation Process}

To make the manual process as efficient as possible, a user interface was created to swiftly click through the images and decide which label is correct. The VGG Image Annotater by Dutta et al. (2016) was used as a base and customized to this specific case. It is a browser-based, simple and lightweight annotation tool which lets the user define and save labels to corresponding pictures in the CSV format. Each image is displayed and the user has to decide if the Google Vision API result, the SwissNames match or neither of them is correct (see Figure 3).

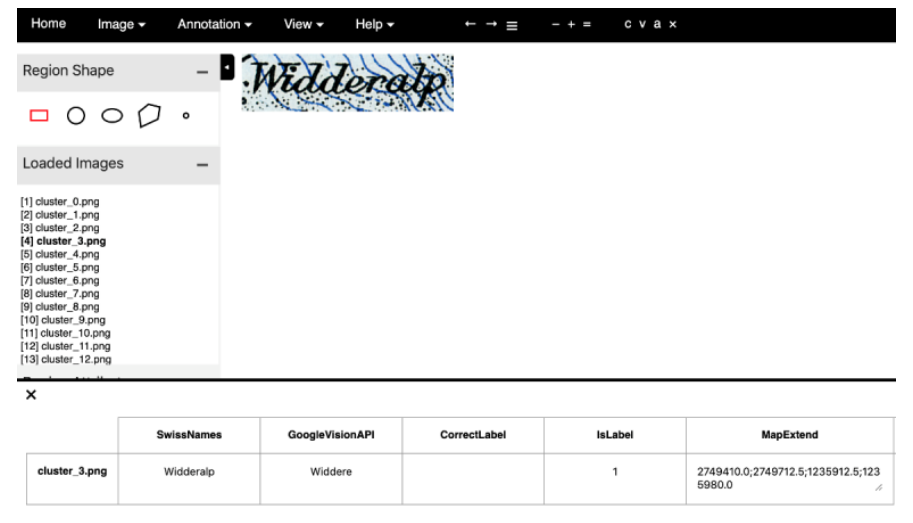

Figure 3. Interface of the adapted VGG Image Annotator

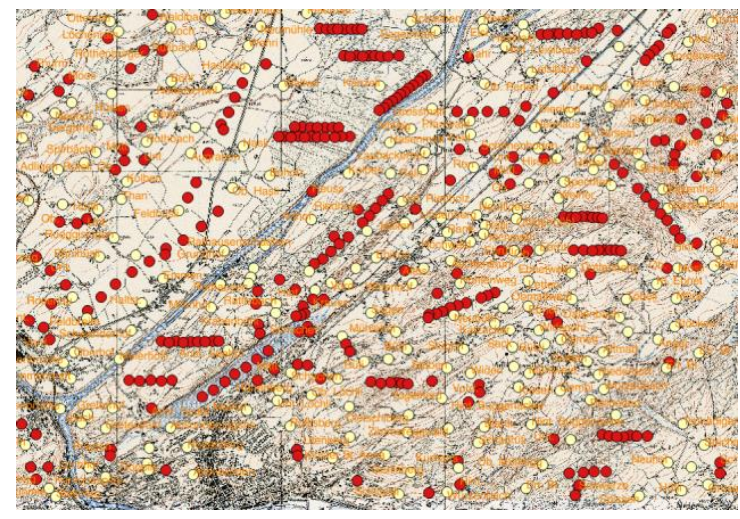

Figure 4. Manual processing in QGIS. Red dots indicate labels which need dome check or correction

Sometimes the clustering algorithm only groups part of the label or several labels together in one cluster. Those cases can be flagged as false and will be corrected in the last step. After the annotation, the results are converted to a shapefile (ESRI, 1998) and loaded into QGIS. There, the incomplete labels are highlighted and can be corrected by placing new labels and filling in the text (see Figure 4). Since the sheets are georeferenced, also the location of the labels can be transformed from the local position to a national coordinate reference system. Once all point features are corrected, the shapefile is saved and the labels of this sheet are successfully digitized. 


\section{Results \& Discussion}

\section{Performance}

The fully automatic part takes around 5-10 min per sheet. It depends on the number of extracted labels, the GPU power and also the internet connection. The usage of the graphics processing unit (GPU) offers the possibility of a high degree of parallelization. If the many simple calculations needed to train and use a neuronal network would have to be done with a CPU, the time would increase drastically.

The manual processing part containing the annotation and post-processing in QGIS takes around 1.5 to 2 hours. The time strongly depends on the quality of the detections as well on the number of labels in the specific area.

Our framework reduces the time consumption of digitizing labels by around a factor of 5 . Compared to a fully manual digitizing process, time efficiency is not the only benefit. Also, the chance of missing labels decreases strongly. We argue that the combination of an automatic procedure followed by a human checking process increases the reliability as well as the accuracy of the detection and classification of labels.

\section{Challenges}

There were several problems we had to cope with during the development of this workflow. The first is the scarcity of training data. The manual segmentation of the labels takes up a huge amount of time and was therefore kept to a minimum. Deep learning models however are famously known for needing a lot of training data. This is probably also the reason why the algorithm struggles with the sheets in alpine regions. The rock hachures look similar to label structures and there are many false positives. When a label is missed in the first step, it can only be found again in the last manual processing step in QGIS.

A second issue shows up in the clustering step. In the French speaking parts of Switzerland almost each label consists of several words. Since the clustering algorithm was customized for German speaking regions, where the labels are often one single word, each word is identified as a single label. This increases the manual processing time since those single words have to be merged to multi-word labels. Ideally, the DBSCAN parameters should be adapted according to the different regions.

When matching with the SwissNames database, we encountered some language based problems. In the Siegfried maps the labels were all written in standard German. Nowadays, all text is written in the local dialect, which can greatly differ to original German spelling. This complicates the matching process. The French spelling also changed in the last 100 years, which always makes another manual intervention necessary to correct the differences.

\section{Outlook}

There are several different ideas on how to go on with the project. Firstly, a straight forward approach would be to generate additional and more diverse training data. This would improve the prediction of segmentation and also make it more reliable. Since by now already 300 of the 500 sheets have been fully processed with the workflow presented here, this data could be used as new ground truth. One idea is to tailor the Google Vision step to this specific data by either 
retraining it or using another text recognition algorithm which is less of a black box and provides more insights and control. Also the clustering algorithm could be extended by adding additional rules or methods to boost performance and capture the reality more accurately.

Of the several versions of each sheet, for now only the latest sheet has been chosen for processing. To find the labels on sheets of other epochs, a comparison with the digitized epoch could help to find the correct label more efficiently.

\section{References}

Bleuer, A. (1968). Zum 100-Jahr-Jubiläum der Siegfriedkarte der Schweiz. Geographica Helvetica, 23, 153-156. https://doi.org/10.5194/gh-23-153-1968

Chiang, Y.-Y. (2017). Unlocking Textual Content from Historical Maps - Potentials and Applications, Trends, and Outlooks. In K. Santosh, M. Hangarge, V. Bevilacqua, \& A. Negi, Recent Trends in Image Processing and Pattern Recognition (Vol. 709, pp. 111-124). Singapore: Springer. https://doi.org/10.1007/978-981-10-4859-3_11

Chiang, Y.-Y., Leyk, S., \& Knoblock, C. A. (2014). A Survey of Digital Map Processing Techniques. ACM Computing Surveys, 47(1). https://doi.org/10.1145/2557423

Deseilligny, M. P., Men, H. L., \& Stamon, G. (1995). Character string recognition on maps, a rotation-invariant recognition method. Pattern Recognition Letters, 16(12), 1297-1310. https://doi.org/10.1016/0167-8655(95)00084-5

Dutta, A., Gupta, A., \& Zissermann, A. (2016). VGG Image Annotator (VIA), 1.0.4. Retrieved Feb 10, 2020, from http://www.robots.ox.ac.uk/ vgg/software/via/

ESRI (1998). ESRI Shapefile Technical Description. ESRI White Paper.

Ester, M., Kriegel, H.-P., Sander, J., \& Xu, X. (1996). A density-based algorithm for discovering clusters a density-based algorithm for discovering clusters in large spatial databases with noise. Proceedings of the Second International Conference on Knowledge Discovery and Data Mining (KDD’96)(AAAI Press), 226-231.

Frischknecht, S. (1996). Extraktion beliebig orientierter Kartensymbole und Schriftelemente aus gescannten topographischen Karten durch rotationsinvariante Filterfunktionen und Template Matching. In L. Hurni, Kartographie im Umbruch - Neue Herausforderungen, neue Technologien (Vol. 96, pp. 180-186). Schweizerische Gesellschaft für Kartographie.

Google (2020). Vision - Derive Vision Insights via ML. Retrieved Feb 1, 2020, from Cloud Vision API: https://cloud.google.com/vision

Heitzler, M., \& Hurni, L. (2020). Cartographic reconstruction of building footprints from historical maps: A study on the Swiss Siegfried map. Transactions in GIS.

https://doi.org/10.1111/tgis.12610

Levenshtein, V. I. (1966, Feb). Binary Codes Capable of Correcting Deletions, Insertions and Reversals. Soviet Physics Doklady, 10, 707.

Ronneberger, O., Fischer, P., \& Brox, T. (2015). U-Net: Convolutional Networks for Biomedical Image Segmentation. In N. a. Navab (Ed.), Medical Image Computing and Computer-Assisted Intervention -MICCAI 2015 (pp. 234-241). Cham: Springer International Publishing. https://doi.org/10.1007/978-3-319-24574-4 28 
Swisstopo (2013, Aug 15). swissNAMES3D. Bundesamt für Landestopografie (Art. 30 GeoIV). Bern.

Velázquez, A., \& Levachkine, S. (2004). Text/Graphics Separation and Recognition in RasterScanned Color Cartographic Maps. In J. Lladós, \& Y. Kwon, Graphics Recognition. Recent Advances and Perspectives. GREC 2003 (Vol. 3088). Berlin, Heidelberg: Springer. https://doi.org/10.1007/978-3-540-25977-0_6

Wick, C., \& Puppe, F. (2018). Fully Convolutional Neural Networks for Page Segmentation of Historical Document Images. 13th IAPR International Workshop on Document Analysis Systems (DAS), 287-292. https://doi.org/10.1109/DAS.2018.39 